\title{
Surface Roughness Prediction in Grinding: a Probabilistic Approach
}

\author{
Krishna Kumar Saxena ${ }^{1}$, Sanjay Agarwal ${ }^{2}$ and Raj Das ${ }^{1, a}$ \\ ${ }^{1}$ Department of Mechanical Engineering, Centre for Advanced Composite Materials, University of Auckland, \\ Auckland 1010, New Zealand, ksax995@aucklanduni.ac.nz r.das@auckland.ac.nz \\ ${ }^{2}$ Department of Mechanical Engineering, B.I.E.T., Jhansi, Uttar Pradesh, India, ${ }^{a}$ sanjay72ag@rediffmail.com
}

\begin{abstract}
Surface quality of machined components is one of the most important criteria for the assessment of grinding processes. The importance of surface finish of a product depends upon its functional requirements. Since surface finish is governed by many factors, its experimental determination is laborious and time consuming. So the establishment of a model for the reliable prediction of surface roughness is still a key problem for grinding. In this study, a new analytical surface roughness model is developed on the basis of the stochastic nature of grinding processes. The model is governed mainly by the random geometry and the random distribution of cutting edges on the wheel surface having random grain protrusion heights. A simple relationship between the surface roughness and the chip thickness was obtained, which was validated by the experimental results using AISI 4340 steel in surface grinding.
\end{abstract}

\section{Introduction}

Advanced engineering materials have been extensively used in industrial applications during the last two decades $[1,2]$. However, the actual utilization of advanced materials with high strength and hardness has been quite limited mainly because of the machining difficulties and associated high cost of machining these materials by grinding whilst ensuring the workpiece quality. A technological basis to achieve more efficient utilization of the grinding process requires an understanding of the interaction between the abrasive and the workpiece, which has direct bearing on the surface roughness, produced using electron discharge machining. Although extensive research [3-11] has been carried out to predict the surface roughness of the ground workpieces, a complete understanding of surface roughness in grinding is yet to be achieved.

Theoretical methods of surface roughness evaluation, as reported in the literature, can be classified into empirical and analytical methods. In the empirical methods, the surface roughness model is developed as a function of kinematic conditions [3-4]. Although the development of empirical models requires less efforts compared to analytical one, they have limited applicability; however, still these models are used in the field of grinding technology. Hence, the scope of these models is limited. The analytical models are always preferred over empirical models as these models are deductively derived from fundamental principles. So, the main advantage of the analytical model is that the results can easily be transferred to different grinding conditions and processes. Hence, these results can be made

\footnotetext{
${ }^{\mathrm{a}}$ Corresponding author : r.das@auckland.ac.nz
} 
applicable to a wide range of process conditions. The analytical models for surface roughness have always been characterized by the description of the microstructure of the grinding wheel, in onedimensional form, taking the grain distance, the width of cutting edge and the grain diameter into account [5-7]. However, these models did not consider the different heights of cutting edges and assumed that the distance between the cutting edges was uniform. Tonshoff et al. [8] described the state of art in the modelling and simulation of grinding processes comparing different approaches to modelling. This work identified a simple basic model where all the parameters such as wheel topography, workpiece material properties and grinding parameters have been lumped into an empirical constant.

Several analytical models, based on the stochastic nature of grinding processes, were proposed [911] to simulate the surface profile generated during grinding. Assuming a particular probability distribution of these random cutting points, output surface profiles were generated for a known input surface profile and input grinding conditions. Experiments conducted by Lal and Shaw [12] with single abrasive grain under fine grinding conditions indicated that the grain tip could be better approximated by a circular arc. Therefore, it is evident that the groove produced by an individual grain can be better approximated by an arc of a circle. Based on this concept, Agarwal and Rao [13-15] developed an analytical model for the prediction of surface roughness in ceramic grinding. A probabilistic approach, for geometric analysis of the grooves generated, was used to describe the surface roughness, in which, the random variable had been defined by undeformed chip thickness probability density function. A simple relationship between the surface roughness and the undeformed chip thickness was found.

In this paper, an analytical model has been envisaged to evaluate surface roughness from the chip thickness probability density function. A simple relationship between the surface roughness and the chip thickness has been established with the chip thickness as a random variable. The model is then verified using experimental data from the surface grinding of AISI 4340 steel with aluminium oxide abrasive.

\section{Model development}

A schematic diagram showing the transformation of the grain tip to the work piece is given in Fig. 1. At any transverse section, the shape of the groove generated by any grain is shown in Fig. 2. Since an individual grain has many tiny cutting points on its surface and the speed ratio is high, the groove produced by an individual grain can be assumed to be an arc of a circle. It can be further assumed that the material is either ploughed with little side pile-up or removed in the form of chips whenever grainwork piece interference occurs.

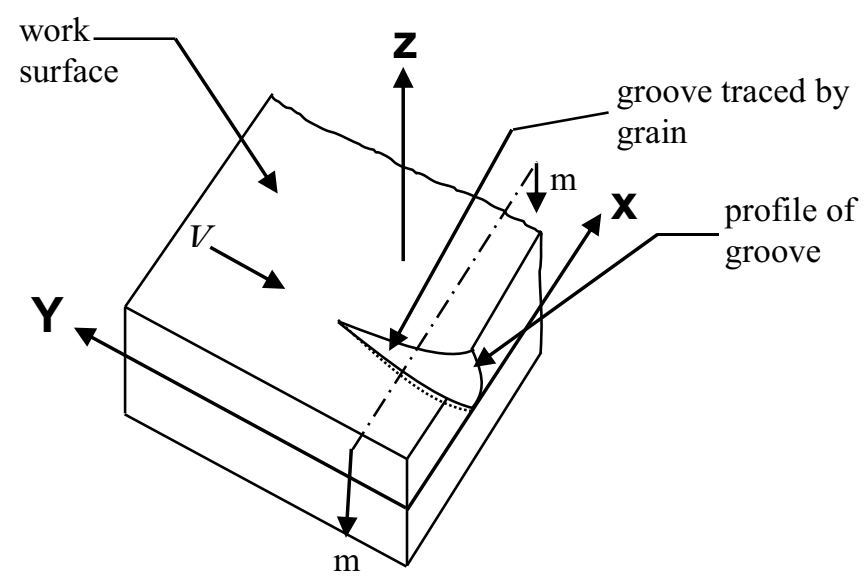

Figure 1. Schematic view of the workpiece in Cartesian coordinate system 


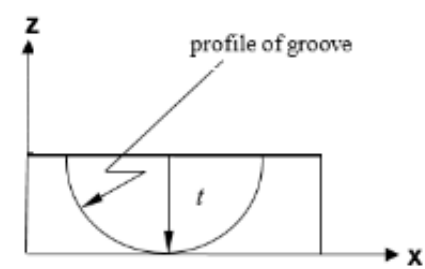

Figure 2. Sectional view showing the shape of groove generated.

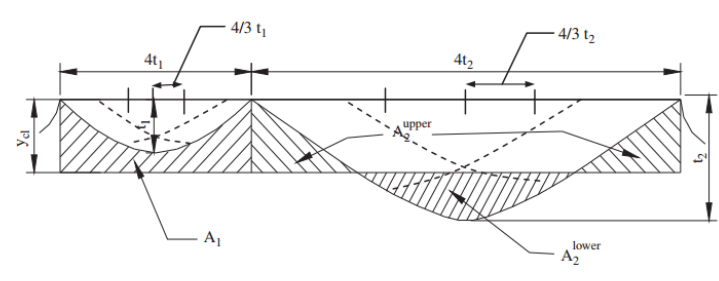

Figure 3. Profile of grooves generated.

Since the position of a grain tip will be random due to random occurrence of grains on the wheel surface, a probability density function is required to describe the surface roughness for all the grains engaged. Thus, the undeformed chip thickness ' $t$ ' can be better described by Rayleigh's probability density function ' $f(t)$ ' [11] as;

$$
\begin{aligned}
& f(t)=\frac{t}{\beta^{2}} \exp \left\{-\frac{1}{2}\left(\frac{t}{\beta}\right)^{2}\right\} \text { for } \mathrm{t} \geq 0 \\
& =0 \quad \text { for } \mathrm{t}<0
\end{aligned}
$$

where $\beta$ is a parameter that completely defines the probability density function and it depends upon the cutting conditions, the direction of grinding wheel and the properties of work piece materials. The expected value and the variance of the above function will be given as where $\beta$ is a parameter that completely defines the probability density function and it depends upon the cutting conditions, the direction of grinding wheel and the properties of work piece materials. The expected value and the variance of the above function will be given as $E(t)=\beta \sqrt{\pi / 2}$ and $\operatorname{sd}(t)=\beta \sqrt{\left(2-\frac{\pi}{2}\right)}$. The surface roughness $R_{a}$ which is defined as the arithmetic average of the absolute values of the deviations of the surface profile height from the mean line within the sampling length $l$. $R a$ can be expressed as

$$
R_{a}=\frac{1}{l} \int_{0}^{l}\left|y-y_{c l}\right| d l
$$

where $y_{c l}$ denotes the centre line drawn in such a way that the areas above and below it are equal. It can also be expressed statistically as

$$
R_{a}=\frac{1}{l} \int_{y_{\min }}^{y_{\max }}\left|y-y_{c l}\right| \cdot p(y) \cdot d y
$$

where $y_{\max }$ and $y_{\min }$ are the lowest and highest peak height of the surface profile and $\mathrm{p}(\mathrm{y})$ is the probability that height $y$ of grain has a particular value y and may be expressed as

$$
p(y)=\lim _{\Delta y \rightarrow 0} \frac{\text { probability }\{y-(1 / 2) \Delta y \leq y \leq y+(1 / 2) \Delta y\}}{\Delta y}
$$

The surface roughness $R_{a}$ can be calculated using probability density function defined in Eq. (1). The complete description of surface generated is very difficult due to the complex behavior of different grains producing grooves because of the random grain-work interaction. Thus, certain assumptions have to be made while predicting the surface roughness. The assumptions are as follows:

(1) The profile of the grooves generated is same and completely defined by the depth of engagement or undeformed chip thickness ' $t$ ' 
(2) There is no groove overlapping

(3) An individual grain has many tiny cutting points in its surface, therefore, for simplicity, the grains are approximated as uniform spheres of diameter $d_{g}(=2 t)$, randomly distributed throughout the wheel volume.

On the average, the expected value of an interference area through a sphere is about one fourth of the area of a circle.

As per definition of surface roughness, the area above and below the centre line must be equal i.e. the total expected area can be written as

$$
E\{A(t)\}=0
$$

The above expression can be represented in terms of the probability density function $f(t)$ as

$$
\int_{0}^{\infty} A(t) \cdot f(t) \cdot d t=0
$$

There are two types of grooves generated depending upon their depth of engagement is either less or greater than centre line $y_{c l}$. For the case when the depth of engagement is less than $y_{c l}$ can be expressed as

$$
E\left\{A\left(t_{1}\right)\right\}=\int_{0}^{y_{c l}} A\left(t_{1}\right) \cdot f(t) \cdot d t,
$$

Similarly, for the groove with depth of engagement is greater than $\mathrm{y}_{\mathrm{cl}}$ can be expressed as

$$
E\left\{A\left(t_{2}\right)\right\}=\int_{y_{c l}}^{\infty} A\left(t_{2}\right) \cdot f(t) \cdot d t
$$

Substituting the values from eq. (4a) and (4b), in eq. (3), equation becomes

$$
\begin{array}{ll} 
& \int_{0}^{y_{c l}} A_{1} f(t) d t+\int_{y_{c l}}^{\infty}\left(A_{2}^{\text {upper }}-A_{2}^{\text {lower }}\right) f(t) d t=0 \\
\text { or, } \quad & p_{1} E\left(A_{1}\right)+p_{2}\left\{E\left(A_{2}^{\text {upper }}\right)-E\left(A_{2}^{\text {lower }}\right)\right\}=0
\end{array}
$$

where $p_{1}$ and $p_{2}$ are the probabilities defined in terms of the chip thickness probability density function $f(t)$ as

$$
\begin{aligned}
& p_{1}=\int_{0}^{y_{c l}} f(t) d t \quad \text { for } t\left\langle y_{c l}\right. \\
& p_{2}=\int_{y_{c l}}^{\infty} f(t) d t \text { for } \mathrm{t}>\mathrm{y}_{\mathrm{cl}}
\end{aligned}
$$

The expected area value, for the groove with depth less than centre line contributing to surface roughness $R_{a}$ can be calculated as 


$$
E\left\{A\left(t_{1}\right)\right\}=\left(\frac{2}{2-\sqrt{3}}\right) E\left(t_{1}\right) y_{c l}-\left(\frac{\pi}{6}-\frac{\sqrt{3}}{4}\right)\left(\frac{2}{2-\sqrt{3}}\right)^{2} E\left(t_{1}^{2}\right)
$$

and the expected value of area, for the groove with depth greater than the centre line contributing to surface roughness $R_{a}$ will be given as

$$
\begin{gathered}
E\left(A_{2}^{\text {upper }}\right)=\left(\frac{2}{2-\sqrt{3}}\right)^{2} E\left(t_{2}^{2}\right) \operatorname{Sin}^{-1}\left(\frac{(2-\sqrt{3}) r}{2 t_{2}}\right)-\left(\frac{\sqrt{3}}{2}\left(\frac{2}{2-\sqrt{3}}\right) t_{2}+y_{c l}\right) r \\
E\left(A_{2}^{\text {lower }}\right)=\left(\frac{2}{2-\sqrt{3}}\right) E\left(t_{2}\right) y_{c l}-\left(\frac{\pi}{6}-\frac{\sqrt{3}}{4}\right)\left(\frac{2}{2-\sqrt{3}}\right)^{2} E\left(t_{2}^{2}\right)+ \\
\left(\left(\frac{2}{2-\sqrt{3}}\right)^{2} E\left(t_{2}^{2}\right) \operatorname{Sin}^{-1}\left(\left(\frac{(2-\sqrt{3}) r}{2 t_{2}}\right)\right)-E\left(\left(\frac{\sqrt{3}}{2}\left(\frac{2}{2-\sqrt{3}}\right) t_{2}+y_{c l}\right) r\right)\right)
\end{gathered}
$$

Where $r=\sqrt{\left\{\frac{t_{2}^{2}}{(2-\sqrt{3})^{2}}-\frac{2 \sqrt{3}}{(2-\sqrt{3})} t_{2} y_{c l}-y_{c l}^{2}\right\}}$ and $E\left(A_{2}^{\text {lower }}\right)$ and $E\left(A_{2}^{\text {upper }}\right)$ are the areas below and above the centre line as shown in Fig. 3. Rewriting the Eq. (6) after substituting the expected values from Eq. (9), (10), (11) as

$$
2 y_{c l}\left\{p_{1} E\left(t_{1}\right)+p_{2} E\left(t_{2}\right)\right\}=\frac{\pi}{2}\left\{p_{1} E\left(t_{1}^{1}\right)+p_{2} E\left(t_{2}^{2}\right)\right\}
$$

To calculate the expected values in the above equation requires another probability density function for the cases where the chip thickness is smaller and greater than the centre line $y_{c l}$. Thus, the conditional probability density function $f_{l}(t)$ and $f_{2}(t)$ defined for each region as

$$
f_{1}(t)=f_{1}\left(t \mid 0 \leq t<y_{c l}\right)=f(t) / \int_{0}^{y_{c l}} f(t) d t
$$

for chip thickness smaller than $\mathrm{y}_{\mathrm{cl}}$ and for rest of the chip thickness

$$
f_{2}(t)=f_{2}\left(t \mid y_{c l} \leq t<\infty\right)=f(t) / \int_{y_{c l}}^{\infty} f(t) d t
$$

Substituting the Eq. (7), (8), (13) and (14) in Eq. (6) to find $y_{c l}$. After simplification the expression for centre line can be expressed as

$$
y_{d}=\left(\frac{\pi}{6}-\frac{\sqrt{3}}{4}\right)\left(\frac{2}{2-\sqrt{3}}\right)\left\{\frac{E\left(t^{2}\right)}{E(t)}\right\}
$$

substituting the expected values in the above equation, and after mathematical simplification, the value of the centre line will be

$$
y_{c l}=\sqrt{\frac{\pi}{2}} \cdot \beta
$$

For the calculation of the surface roughness, both types of grooves are required. Since the contribution of two types of grooves is different, thus, the total expected value of surface roughness can be calculated as; 


$$
E\left(R_{a}\right)=p_{1} E\left(R_{a 1}\right)+p_{2} E\left(R_{a 2}\right)
$$

Where $E\left(R_{a 1}\right)$ and $E\left(R_{a 2}\right)$ are the expected value of the surface roughness for depth of engagement smaller or greater than $y_{c l}$. Thus, the values of the expected values can be calculated by the definition of the surface roughness according to which the surface roughness can be calculated by adding the area between the profile and the centre line and divide it by the total profile length. Hence from Fig. 3, the values can be written as

$$
\begin{gathered}
E\left(R_{a 1}\right)=E\left(\frac{A_{1}}{2 t_{1}}\right) \\
E\left(R_{a 2}\right)=E\left(\frac{A_{2}^{\text {upper }}+A_{2}^{\text {lower }}}{2 t_{2}}\right)
\end{gathered}
$$

Rewriting the Eq. (18) \& (19) after substituting the expressions of $\mathrm{A}_{1}, A_{2}^{\text {upper }} \& A_{2}^{\text {lower }}$ from Eq. (9), (10) and (11) as

$$
\begin{gathered}
E\left(R_{a 1}\right)=y_{c l}-\left(\frac{\pi}{6}-\frac{\sqrt{3}}{4}\right)\left(\frac{2}{2-\sqrt{3}}\right)^{2} E\left(t_{1}\right) \\
E\left(R_{a 2}\right)=y_{c l}-\left(\frac{\pi}{6}-\frac{\sqrt{3}}{4}\right)\left(\frac{2}{2-\sqrt{3}}\right) E\left(t_{2}\right)+2\left(\left(\frac{2}{2-\sqrt{3}}\right) E\left(t_{2}\right) \operatorname{Sin}^{-1}\left(\frac{(2-\sqrt{3}) r}{2 t_{2}}\right)\right) \\
-E\left(\left(\frac{\sqrt{3}}{2}\left(\frac{2}{2-\sqrt{3}}\right) t_{2}+y_{c l}\right)\left(\frac{2-\sqrt{3}}{2}\right) t_{2} r\right)
\end{gathered}
$$

Substituting the expected values of $E\left(t_{1}\right), E\left(t_{2}\right)$ from Eq. (8), (10), (13) and (16) in Eq. (20), (21) and then from eq. (17), the expected value of surface roughness can be expressed as

$$
E\left(R_{a}\right)=1.16 \beta
$$

It can also expressed also in terms of the chip thickness expected value $E(t)$ by replacing $\beta$ in terms of $E(t)$, as

$$
E\left(R_{a}\right)=0.92 E(t)
$$

Eq.(23) shows a proportional relationship between the surface roughness and the chip thickness expected values under the assumption that the profile of grove generated by an individual grain to be a semi-circular in shape and the phenomena like back transferring of material, grain overlapping are absent.

\section{Prediction of surface roughness}

The model has to be evaluated to determine its validity and versatility. The validity of the model is assessed through a comparison between the predicted value and measured value of the surface roughness within the predefined range of parameters. The versatility is based upon the usefulness of the model in predicting the kinematic conditions which would be required in order to produce specified surface finish. In order to compare the theoretical results obtained by making use of the model developed, experiments has been conducted in the horizontal surface grinding configuration. The work material taken was AISI 4340 steel with hardness 65HRC. The amount of material removed 
was the same for each experiment to maintain a consistent state of the wheel surface. The other conditions taken for experiment purpose are as follows; wheel speed $36.6 \mathrm{~m} / \mathrm{sec}$, wheel diameter 250 $\mathrm{mm}$, wheel width $19 \mathrm{~mm}$. The kinematic parameters for each experiment are depth of cut $\left(a_{e}\right)$ and the speed ratio $\left(v_{s} / v_{w}\right)$ where $v_{w}(5,10$ and $15 \mathrm{~m} / \mathrm{min})$ is the feed rate and $v_{s}$ is the wheel speed, as shown in Table 1.

Table 1 Experimental Conditions

\begin{tabular}{|l|ccccccccc|}
\hline Exp. No. & 1 & 2 & 3 & 4 & 5 & 6 & 7 & 8 & 9 \\
\hline $\mathrm{a}_{\mathrm{e}(\mu \mathrm{m})}$ & 5 & 5 & 5 & 10 & 10 & 10 & 15 & 15 & 15 \\
\hline $\mathrm{V}_{\mathrm{s}} / \mathrm{v}_{\mathrm{w}}$ & 440 & 220 & 146 & 440 & 220 & 146 & 440 & 220 & 146 \\
\hline $\mathrm{R}_{\mathrm{a}}(\mu \mathrm{m})$ & 0.184 & 0.271 & 0.329 & 0.206 & 0.297 & 0.367 & 0.220 & 0.331 & 0.394 \\
\hline
\end{tabular}

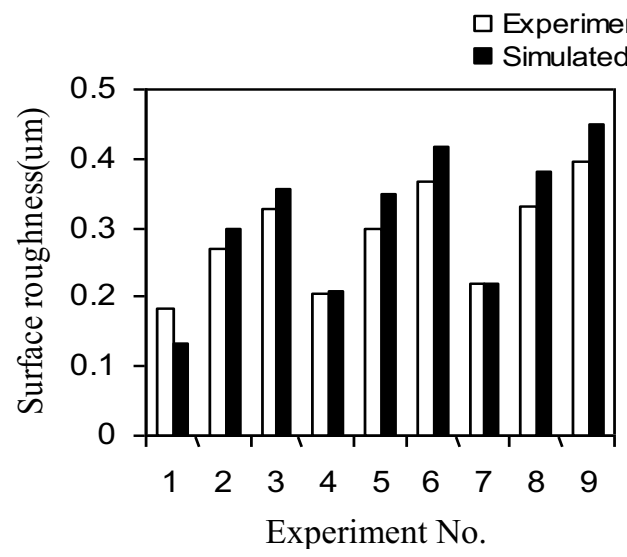

Figure 4. Experimental and simulated values of surface roughness for surface grinding.

Surface roughness measurements were made using Talysurf-VI (cut-off length was $0.8 \mathrm{~mm}$ ) at five different places on the $20 \times 5 \mathrm{~mm}^{2}$ cross-section of the workpiece after grinding and the arithmetic mean of the values of the measurements has been reported in the experimental results as shown in Table 1. The experiments are replicated five times (as shown in Table 1) to mask the variability of the process. By making use of the model of chip thickness developed in [16], the chip thickness expected value was calculated for each experiment.

To validate the model, the centre line average of surface roughness of all ground work pieces of the each set of experiment after a single pass was obtained using Talysurf-6 surface roughness measuring instrument. The results are plotted along with the experiment values of the surface roughness as shown in Fig. 4. It can be easily observed that the predicted value of the surface roughness shows a good agreement with the experimental data obtained from the different kinematic conditions in horizontal surface grinding.

\section{Results and discussion}

The number of variables that influence the surface roughness of ground surfaces are numerous. These include the wheel, work and machine characteristics and the operating characteristics. Since the surface roughness model developed above is based on the chip thickness model which includes many parameters such as kinematic conditions, material properties etc. Therefore, this model can be used to calculate the surface roughness under different conditions of these parameters. The most common kinematic parameters are depth of cut and the speed ratio. Further the deviation of the surface roughness of the new models from the actual values, for various feeds and depth of cut is shown in Fig. 5. Typical results showing the effect of the grinding conditions on the surface roughness. It is seen 
that with increase in the values of feeds and depth of cut, surface-finish deteoriates. This is probably due to the forces generated at higher feeds and depth of cut that results in higher deflections.
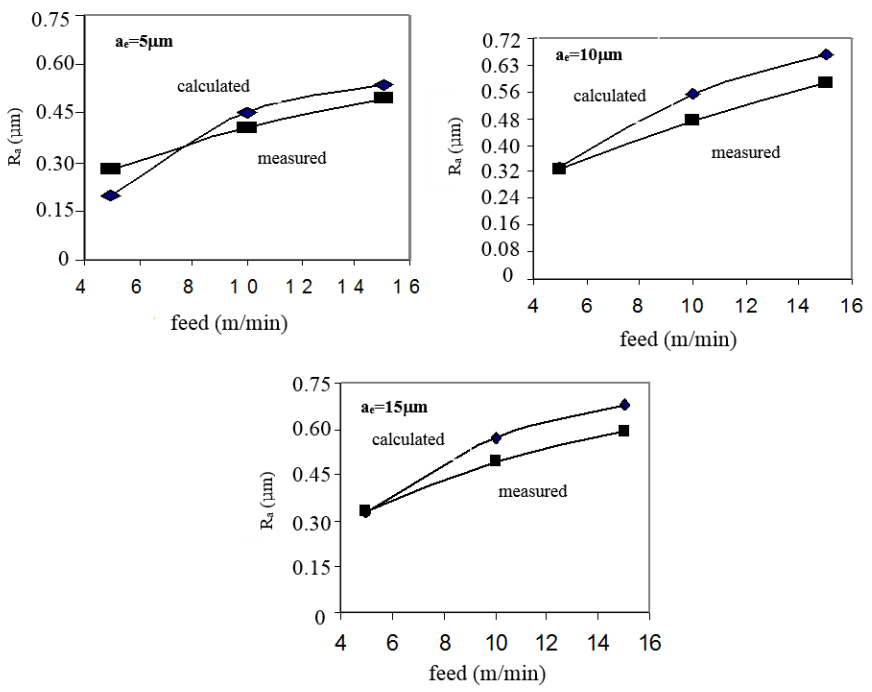

Figure 5. Surface roughness presented against feed at various depths of cut.

Also, the value of surface roughness is slightly higher than the measured value for various values of feeds and depth of cut. The probable reason for this is the assumption that the profile of grooves generated is perfectly semi-circle which is not always the case. It can be observed from the Fig. 5 that the maximum deviation in the prediction of surface roughness with chip thickness model is about 1.15 times from the actual value. Another important parameter that will have significant effect on the surface roughness is the undeformed chip thickness. With increasing chip thickness, surface roughness will increase. Thus it appears that the chip thickness has pronounced effect causing a deteoriation of surface finish. This aspect is indicated by both theoretical and experimental results as shown in Fig. 4. Further, there appears to be reasonably close agreement between the experimental and simulated results.

\section{Conclusions}

In this paper, a simplified model is proposed for predicting the surface roughness of a work piece during the grinding process, taking into consideration the random distribution of the grain protrusion heights and assuming the profile of a groove generated by an individual grain to be an arc of a circle. The model is based on the relationship between the variables of the surface roughness and the undeformed chip thickness. The variables such as wheel microstructure, the kinematic and dynamic grinding conditions, and the material properties were included in the model through undeformed chip thickness model.The predicted surface roughness appears to yield results which agree reasonably well with the experimental values for different kinematic conditions in the horizontal surface grinding of AISI 4340 steel. Thus, the analytical model developed above is established to be a reliable and efficient technique for the prediction of surface roughness.

\section{References}

1. S. Agarwal, P.V. Rao, Int. J.Mach. Tools Mf. 50-12, 1077 (2010)

2. S. Agarwal, P.V. Rao, Int. J.Mach. Tools Mf. 48-6, 698 (2008) 
3. S. Malkin, Grinding Technology: Theory and Applications of Machining with Abrasives (Ellis Horwood, Chichester, West Sussex, 1989)

4. T. Suto, T. Sata, Bull. Jap. Soc. Prec. Engng. 15-1, 27 (1981)

5. K. Sato, Reports of Tokohu University, 20-1, 59 (1955)

6. T. Orioka, Bull. Japan Soc. Grinding Engrs, 27 (1961)

7. G.K. Lal, M.C. Shaw, Trans. ASME, J. Engng for Industry. 1119 (1975)

8. H. Tonshoff, J. Peters, I. Inasaki, T. Paul, Ann. CIRP. 41-2, 677 (1992)

9. X. Zhou, F.Xi, Int. J. Mach. Tools Mf. 42, 969 (2002)

10. S.S. Law, S.M. Wu, Trans. ASME, J. Engng for Industry. 95, 972 (1973)

11. R.L. Hecker, S. Liang, Int. J. Mach. Tools Mf. 43, 755 (2003)

12. G.K. Lal, M.C. Shaw, Proc. Int. Grindng Conf., Carnegie-Mellon University, Pittsburgh, USA. 107 (1972)

13. S. Agarwal, P.V. Rao, Int. J. Mach. Tools Mf. 45-6, 609 (2005)

14. S. Agarwal, P.V. Rao, Proc. of IMechE, Part B, J. Eng. Mf. 219, 811 (2005)

15. S. Agarwal, P.V. Rao, Int. J. Mach. Tools Mf. 50-2, 1035 (2010)

16. S. Agarwal, P.V. Rao, Int. J. Mach. Tools Mf. 56, 59 (2012) 